\title{
A ROUGHNESS AND TIME DEPENDENT MIXING LENGTH EQUATION
}

\author{
Rafik ABSI \\ Assoc. Professor, EBI, Inst. Poly. St-Louis, \\ and Assoc. Researcher, Laboratoire Roberval, UTC-CNRS. \\ (EBI, 32 Bd du Port, 95094 Cergy-Pontoise cedex, France) \\ E-mail: r.absi@ebi-edu.com
}

\begin{abstract}
A roughness and time dependent mixing length equation is developed from an extension of von Kármán's similarity hypothesis, based on local equilibrium (turbulent energy production balanced by the dissipation), with an algebraic equation for the shape of the turbulent kinetic energy. Our vertical mixing length profile and the related mean velocities approach the experimental data well. We show that our equation follows Prandtl's mixing length equation only near a smooth wall. The use of the proposed timedependent mixing length equation in a turbulent oscillatory boundary layer shows, like the $k-\varepsilon$ model, an increase in the mixing length and the eddy viscosity near flow reversal.
\end{abstract}

Key words: mixing length, vertical profile, roughness and time dependent, eddy viscosity, mean velocity, oscillatory flow reversal

\section{INTRODUCTION}

The development of theoretical and semitheoretical analytical methods for turbulent flows is of great importance in both practical engineering applications and basic turbulence research. The mechanism of turbulence is of an extremely complicated nature. A turbulent flow that presents fluctuations involves a complicated formulation of momentum equations. In these flows, the turbulent mixing motion is responsible not only for an exchange of momentum, but it also enhances the transfer of heat and mass in the flow. For equilibrium flows, where the turbulent energy production is balanced by the dissipation, it is easy to find the Prandtl's mixing length hypothesis with a proportional relation between the turbulent length scale and the mixing length.

Even if the notion of mixing length $l$ is important, it is still related to Prandtl's mixing length equation $l=\kappa \cdot y$, which presents limitations since it is linear, roughness and time independent. It is important therefore to develop a more general mixing length equation that is non-linear, roughness and time dependent. The mixing length formulation based on an extension of von Kármán's similarity hypothesis seems to be a suitable tool for developing an algebraic roughness and time dependent mixing length equation. In this paper, we will present a semi-theoretical algebraic equation for the mixing length together with the related assumptions. The vertical and timedependent profiles will be validated.

\section{BACKGROUND}

Near a wall, the turbulent length scale is estimated to be proportional to the size of the large eddies, those that contain the most energy, and thus the most momentum. From a certain distance $y$, if we assume that the most effective eddies for the mixing are precisely those of size $y$, we can write $l \sim y$ which gives Prandtl's mixing length equation:

$$
l=\kappa y
$$

where $\kappa$ is the von Kármán's constant $(\kappa \approx 0,4)$. In a turbulent boundary layer, the largest eddies are 
limited by the transverse dimension of the flow namely the boundary layer thickness $\delta$, we are therefore able to write

$$
l \approx \delta
$$

Indeed, Escudier ${ }^{1)}$ wrote the mixing length as:

$$
\frac{l}{\delta}=\left\{\begin{array}{ccc}
K \frac{y}{\delta} & \text { for } & \frac{y}{\delta} \leq \frac{\lambda}{K} \\
\lambda & \text { for } & \frac{y}{\delta}>\frac{\lambda}{K}
\end{array}\right.
$$

where: $K=0,43 \quad, \quad \lambda=0,09$.

We write the eddy viscosity from the mixing length $l$ and the turbulent kinetic energy $k$ as:

$$
v_{t}=\sqrt{C_{v}} l \sqrt{k}
$$

where $C_{v}$ is a constant, $\left(C_{v} \approx 0.3\right.$ from experiments in boundary layers and open-channel flows). In local equilibrium region, where the energy production is balanced by the dissipation, we are able to find the mean velocity profile from the mixing length $l$ and the turbulent kinetic energy $k$ by:

$$
\frac{d U}{d y}=\sqrt{C_{v}} \frac{\sqrt{k}}{l}
$$

where $\mathrm{U}$ is the mean flow velocity. The assumption of a parabolic shape for the eddy viscosity:

$$
v_{t}(y)=\kappa U_{f} y\left(1-\frac{y}{h}\right),
$$

( $U_{f}$ is the friction velocity and $h$ the scale of the flow, the boundary layer thickness or the flow depth) and a logarithmic profile for the mean flow velocity:

$$
U(y)=\frac{U_{f}}{\kappa} \ln \left(\frac{y}{y_{0}}\right),
$$

( $y_{0}$ is the hydrodynamic roughness) imply a mixing length equation in the form:

$$
l=\kappa y \sqrt{1-\frac{y}{h}}
$$

Indeed, from (4) and (5), we are able to write $\frac{d U}{d y}=\frac{v_{t}}{l^{2}}$ and therefore $l=\sqrt{\frac{v_{t}}{\frac{\partial U}{\partial y}}}$; with (6) and

(7), we obtain therefore (8). From (4), (6) and (8), the corresponding turbulent kinetic energy $k$ profile is given by:

$$
\sqrt{k}=\frac{1}{\sqrt{C_{V}}} U_{f} \sqrt{1-\frac{y}{h}}
$$

This shape for $k$, verifies the logarithmic velocity profile $^{2)}$ since (5), (8) and (9) give $\frac{d U}{d y}=\sqrt{C_{V}} \frac{\sqrt{k}}{l}=\frac{U_{f}}{\kappa y}$ and therefore (7).

On the other hand, the assumption of a parabolic shape for the eddy viscosity (6) and a linear mixing length profile (1), imply a turbulent kinetic energy $k$ in the form:

$$
\sqrt{k}=\frac{1}{\sqrt{C_{V}}} U_{f}\left(1-\frac{y}{h}\right)
$$

This profile gives a non-logarithmic velocity profile, since:

$$
\frac{d U}{d y}=\sqrt{C_{v}} \frac{\sqrt{k}}{l}=\frac{U_{f}\left(1-\frac{y}{h}\right)}{\kappa y}
$$

\section{METHODOLOGY}

The von Kármán's similarity hypothesis ${ }^{3)}$, which assumes that turbulent fluctuations are similar at all points of the field of flow (similarity rule), gives the mixing length in the form:

$$
l=-\kappa\left[\frac{\left(\frac{\partial U}{\partial y}\right)}{\left(\frac{\partial^{2} U}{\partial y^{2}}\right)}\right]
$$

For local equilibrium (5), an extension of von Kármán's similarity hypothesis allows to write:

$$
l=-\kappa\left[\frac{\frac{\sqrt{k}}{l}}{\frac{\partial}{\partial y}\left(\frac{\sqrt{k}}{l}\right)}\right]
$$

We can write (13) in the form: $-\frac{f^{\prime}}{f^{2}}=\frac{\kappa}{\sqrt{k}}$, with: $f=\frac{\sqrt{k}}{l} ;$ applying the condition $l\left(y=y_{0}\right)=\kappa y_{0} \quad$ and $\quad$ by integrating $\int_{y_{0}}^{y}-\frac{f^{\prime}}{f^{2}} d y=\frac{1}{f}-\frac{1}{f_{0}}=\kappa \int_{y_{0}}^{y} 1 / \sqrt{k} d y$, we obtain:

$$
l=\kappa \sqrt{k}\left[\int_{y_{0}}^{y} 1 / \sqrt{k} d y+\frac{y_{0}}{\sqrt{k_{0}}}\right]
$$

where $k_{0}=k\left(y_{0}\right)$. Equation (14) can be integrated if the turbulent kinetic energy is given by an algebraic equation. 


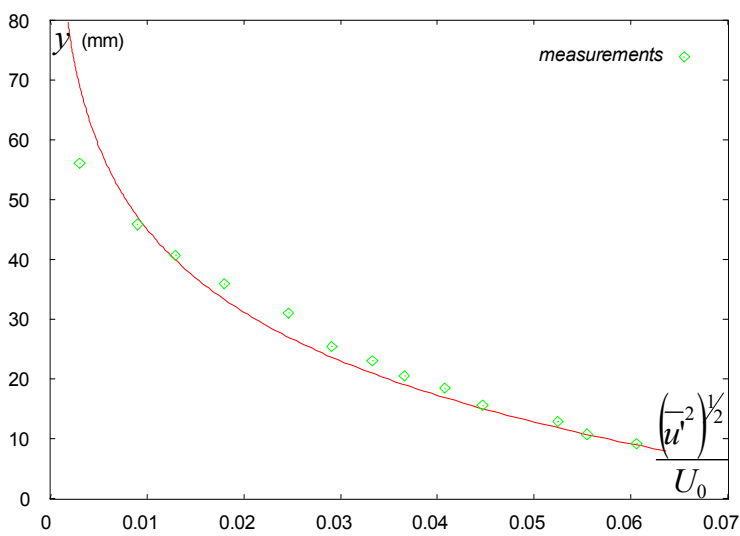

Fig.1 Variation of turbulence intensity with height; $\diamond$ : measurements ${ }^{6)}$, the curve is obtained from equation (15).

\section{(1) A more accurate algebraic equation for the} turbulent kinetic energy

Determining algebraic equations for turbulence intensities and the turbulent kinetic energy is important in both practical applications and basic turbulence research. In this study, we assume that: $v^{\prime}=C_{v} u^{\prime}$ and $w^{\prime}=C_{w} u^{\prime}$, with $C_{v}$ and $C_{w}$ are constants in the energy equilibrium region ${ }^{4)}$, we can therefore write: $\sqrt{k} \approx\left(\overline{u^{12}}\right)^{\frac{1}{2}}$. For uniform clearwater open channel flow the turbulence intensity profiles have been extensively investigated. The experiments in clear water flows of Song et al. ${ }^{5}$ confirmed that for rough beds, the dimensionless turbulence intensity could be expressed by a function, which decreases exponentially with y.

From turbulence intensity measurements ${ }^{6}$ over mobile bed, we noticed ${ }^{7)}$ that $\left(\overline{u^{12}}\right)^{\frac{1}{F}}$ can be expressed by an exponential function, which decreases with y (Fig. 1):

$$
\left(\overline{u^{\prime 2}}\right)^{\frac{1}{2}}=\text { const. } U_{f} \exp \left(-c_{1} y / h\right)
$$

Therefore, we wrote $\sqrt{k}$ as:

$$
\sqrt{k}=c_{2} \cdot U_{f} \exp \left(-c_{1} y / h\right)
$$

$c_{1}$ and $c_{2}$ are two parameters, with $c_{2}=1 / \sqrt{C_{V}}$.

On the other hand, for steady open channel flows in local equilibrium, from the modeled k-equation, Nezu and Nakagawa ${ }^{4)}$ obtained: $k / U_{f}^{2}=D \exp \left(-2 C_{k} y / h\right)$, (where: $\mathrm{C}_{\mathrm{k}}$ and $\mathrm{D}$ are empirical constants) and therefore equation (16) for $\sqrt{k}$.

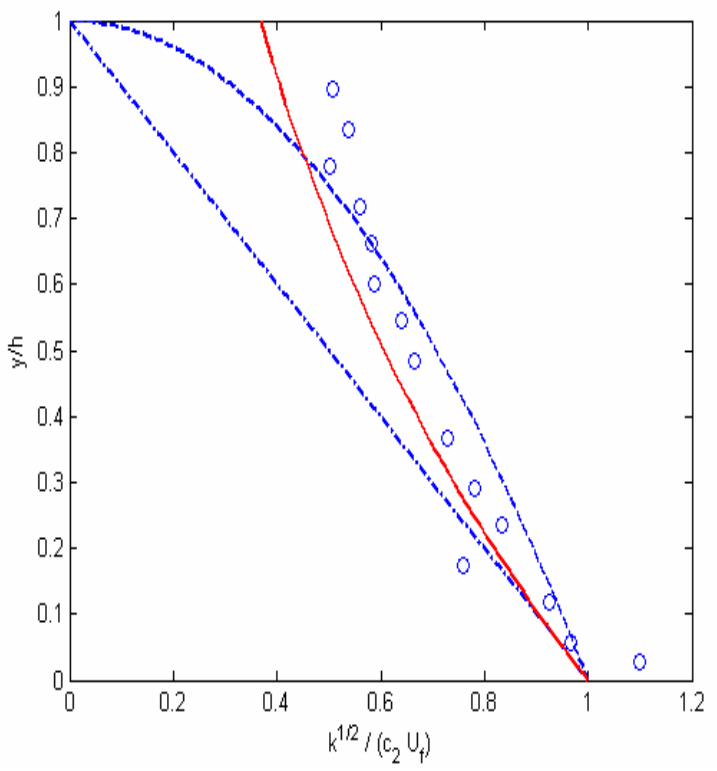

Fig.2 Comparison between the different profiles for the turbulent kinetic energy $k$; dashed line: equation (9); dash-dotted line: equation (10); solid line: equation (16) with $c_{1}=1$, Symbols: measurements ${ }^{8)}$ for profile 5 .

Fig. (2) shows a comparison between the different turbulent kinetic energy profiles obtained from equations (9), (10) and (16) and measurements ${ }^{8}$. This figure demonstrate that the profile (16) is more accurate (solid line) than (9) and (10).

From equations (5) and (16), the logarithmic velocity profile hypothesis implies a mixing length equation in the form:

$$
l=\kappa y \exp \left(-c_{1} \frac{y}{h}\right)
$$

since (16) and (17) give $\frac{d U}{d y}=\sqrt{C_{V}} \frac{\sqrt{k}}{l}=\frac{U_{f}}{\kappa y}$ and therefore (7).

\section{(2) A roughness-dependent mixing length equation}

Inserting (16) into (14) and by integrating, we obtain $^{9), 10)}$ :

$$
\begin{aligned}
& l(y)=\kappa \exp \left(-c_{1} y / h\right)\left[h / c_{1} \exp \left(c_{1} y / h\right)\right. \\
& \left.-h / c_{1} \exp \left(c_{1} y_{0} / h\right)+y_{0} \exp \left(c_{1} y_{0} / h\right)\right]
\end{aligned}
$$

We can simplify our mixing length equation (18) in the form:

$$
l(y)=\kappa \cdot\left[h / c_{1}-\left(h / c_{1}-y_{0}\right) \cdot \exp \left(-c_{1} \frac{\left(y-y_{0}\right)}{h}\right)\right]
$$


Table 1 Summary of the different eddy viscosity models

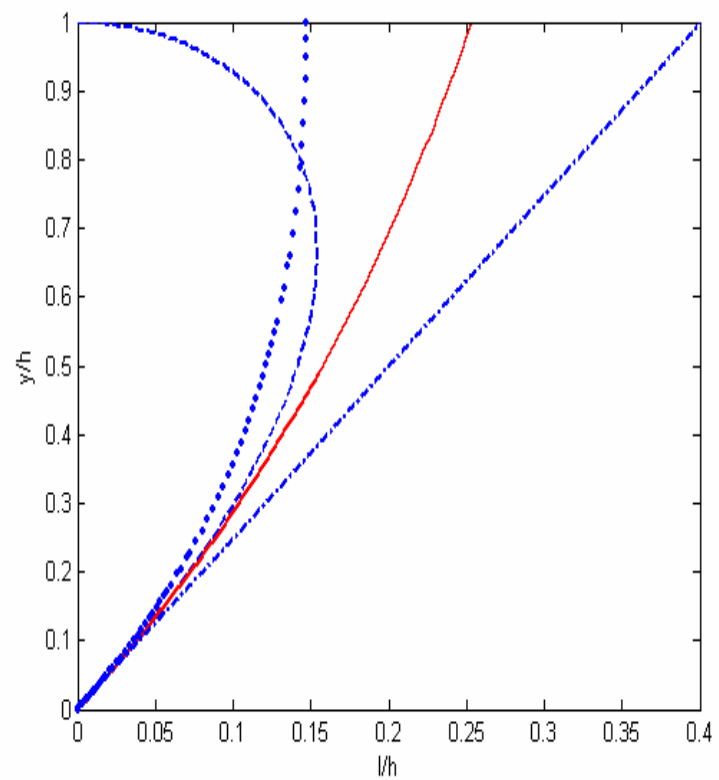

Fig.3 Comparison between the different mixing length profiles; dash-dotted line: equation (1) ; dashed line equation (8); dotted line equation (17); solid line equation (20); with $\mathrm{c}_{1}=1$.

For a smooth wall $\left(y_{0}=0\right)$, we write equation (19) as:

$$
\frac{l(y)}{h}=\frac{\kappa}{c_{1}} \cdot\left[1-\exp \left(-c_{1} \frac{y}{h}\right)\right]
$$

Fig. (3) presents a comparison between mixing length profiles obtained from equations (1), (8), (17) and (20). The four corresponding eddy viscosity models are summarized in Table $\mathbf{1}$.

\section{(3) A time dependent mixing length equation}

Equation (6) was used by Fredsøe et al. ${ }^{11)}$ in oscillatory boundary layers. They took a timedependent eddy viscosity, which is parabolic in the wave boundary layer:

$$
v_{t}(y, t)=\kappa U_{f}(t) y\left(1-\frac{y}{\delta(t)}\right)
$$

In this equation, the friction velocity $U_{f}(t)$ and the boundary layer thickness $\delta(t)$ were found by application of the momentum integral method.

From the analysis of experimental data ${ }^{12)}$, we noticed that the Reynolds stress could be expressed as $^{13), 7)}$ :

$$
-\overline{u^{\prime} v^{\prime}}(y, t) \approx \chi(t) f(y, \zeta(t))
$$

We noticed that $\chi(t) \approx U_{f}^{2}(t)$ and $\zeta(t) \approx \delta(t)$. We wrote therefore the eddy viscosity as:

$$
v_{t}(y, t)=\kappa y U_{f}(t) f_{*}(y, \delta(t)),
$$

which was expressed in a more general form as ${ }^{9)}$ :

\begin{tabular}{|l|l|l|l|}
\hline Eq. (4) & $l$ & $\sqrt{k}$ & $U$ \\
\hline $\begin{array}{l}\text { Model } \\
1\end{array}$ & $\begin{array}{l}\text { Equation } \\
(1)\end{array}$ & $\begin{array}{l}\text { Equation } \\
(10)\end{array}$ & $\begin{array}{l}\text { Non } \\
\text { logarithmic }\end{array}$ \\
\hline $\begin{array}{l}\text { Model } \\
2\end{array}$ & $\begin{array}{l}\text { Equation } \\
(8)\end{array}$ & Equation (9) & Logarithmic \\
\hline $\begin{array}{l}\text { Model } \\
3\end{array}$ & $\begin{array}{l}\text { Equation } \\
(17)\end{array}$ & $\begin{array}{l}\text { Equation } \\
(16)\end{array}$ & Logarithmic \\
\hline $\begin{array}{l}\text { Model } \\
4\end{array}$ & $\begin{array}{l}\text { Equation } \\
(19)\end{array}$ & $\begin{array}{l}\text { Equation } \\
(16)\end{array}$ & $\begin{array}{l}\text { Non } \\
\text { logarithmic }\end{array}$ \\
\hline
\end{tabular}

$$
v_{t}(y, t)=g\left(U_{f}(t)\right) f_{*}(y, \delta(t)) l(y, \delta(t)),
$$

in this equation $f_{*}=\sqrt{f}$ and $g\left(U_{f}(t)\right)$ is the entropy correction function ${ }^{7}$ :

$$
g\left(U_{f}(t)\right)=\left\{\begin{array}{cccc}
\frac{U_{f}^{2}+\varepsilon_{*}^{2}}{2 \varepsilon_{*}} & \text { if } & U_{f} \leq \varepsilon_{*} \\
\left|U_{f}\right| & \text { if } & U_{f} \geq \varepsilon_{*}
\end{array}\right.
$$

where: $\varepsilon_{*}=0.746\left(f_{w} / 2\right)^{1 / 2} U_{0 m} ;\left(U_{0 m}\right.$ is the maximum value of the free stream velocity and $f_{w}$ is the wave friction factor).

From (4) and (24), $\sqrt{k}$ is therefore given by:

$$
\sqrt{k}(y, t)=c_{2} g\left(U_{f}(t)\right) f_{*}(y, \delta(t))
$$

The analysis of experimental data of turbulence intensity in oscillatory flows ${ }^{14),}{ }^{12), 6)}$ allows us to write for very rough and mobile beds a generalization of equation (16) to a time-dependent equation:

$$
\sqrt{k}(y, t)=c_{2} \cdot g\left(U_{f}(t)\right) \exp \left(-c_{1} y / \delta(t)\right)
$$

The function $f_{*}(y, \delta(t))$ is therefore equal here to $\exp \left(-c_{1} y / \delta(t)\right)$. By inserting (26) into (14) and by integrating, we obtain a time-dependent mixing length equation:

$$
\begin{gathered}
l(y, t)=\kappa \exp \left(-c_{1} y / \delta(t)\right)\left[\delta(t) / c_{1} \exp \left(c_{1} y / \delta(t)\right)\right. \\
\left.-\delta(t) / c_{1} \exp \left(c_{1} y_{0} / \delta(t)\right)+y_{0} \exp \left(c_{1} y_{0} / \delta(t)\right)\right]
\end{gathered}
$$

\section{PARTICULARITIES AND INTEREST OF THE PROPOSED EQUATIONS}

\section{(1) The vertical mixing length profile}

We will first analyze the vertical profile of the proposed equation (19). We will consider here the case where $h$ represents the boundary layer thickness $\delta$. 


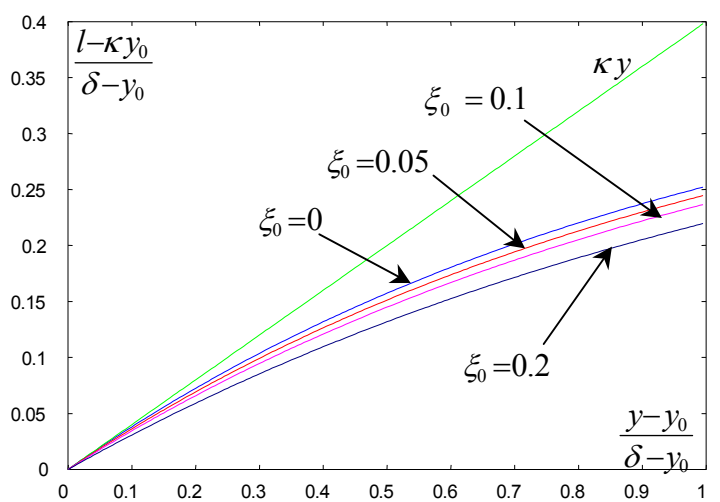

Fig.4 Effect of relative roughness on our mixing length profile $l(y)$ obtained from equation (19) for $c_{1}=1$.

Fig. (4) shows the effect of the relative roughness $\xi_{0}=\left(c_{1} y_{0}\right) / \delta$ on our mixing length profile (19). The difference from the linear equation (1) increases with $\mathrm{y}$, but also with the roughness, different relative roughness are presented. We notice that from a same (imposed) value $\kappa \cdot y_{0}$ in $y_{0}$, our mixing length (19) increases more slowly with $\mathrm{y}$, the gradient $\mathrm{d} l / \mathrm{d} y$, which is equal to $\kappa \cdot\left(1-\xi_{0}\right) \cdot \exp \left(-\left(\xi-\xi_{0}\right)\right)$, is everywhere (since $\left.y \geq y_{0}\right)$ smaller than $\kappa$, where $\xi=\left(c_{1} y\right) / \delta$. Moreover, the slope at the origin $y_{0}$, equal to $\kappa \cdot\left(1-\xi_{0}\right)$, decreases with the roughness $y_{0}$ (Fig. 4); this involves the introduction of an effective kappa $\kappa^{*}$ as:

$$
\kappa^{*}=\kappa \cdot\left(1-\xi_{0}\right)
$$

The difference $\kappa^{*} \neq \kappa$ shows that even at the first order (linearization on the parameter $\left(\xi-\xi_{0}\right)$ which is assumed to be small, near the wall), our profile is different from the Prandtl's profile $\kappa \cdot y$. It is written as:

$$
l(y) \approx \kappa^{*} \cdot y+\kappa \cdot y_{0} \xi_{0}
$$

The two profiles are different even near the wall ${ }^{16)}$. They will be identical in the sole case of low roughness (smooth wall). Only here, $\kappa^{*} \approx \kappa$ and $l(y) \approx \kappa \cdot y$. We can conclude that equation (1) is able to describe the vertical profile of mixing length near the wall and only for smooth walls.

For a smooth wall ( $\left.\xi_{0}=0\right)$, Fig. 5.a) shows that the mixing length profile (equation 20), which is non-linear, is different from Prandtl's profile (equation 1). A comparison between our proposed profile and experimental data is presented in Fig. 5.

Fig. 5. b) presents experimental data and mixing a)

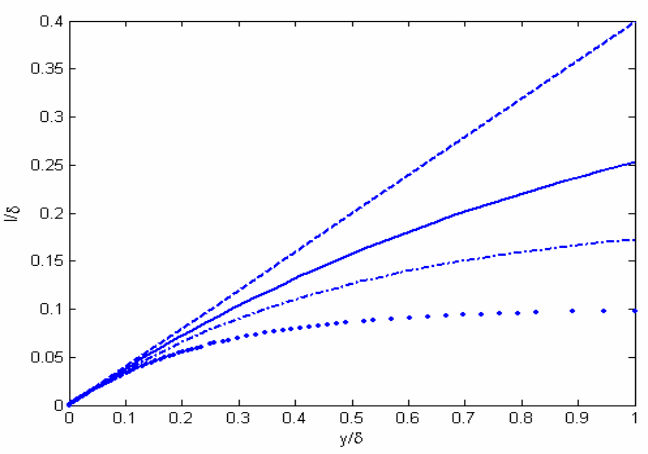

b)

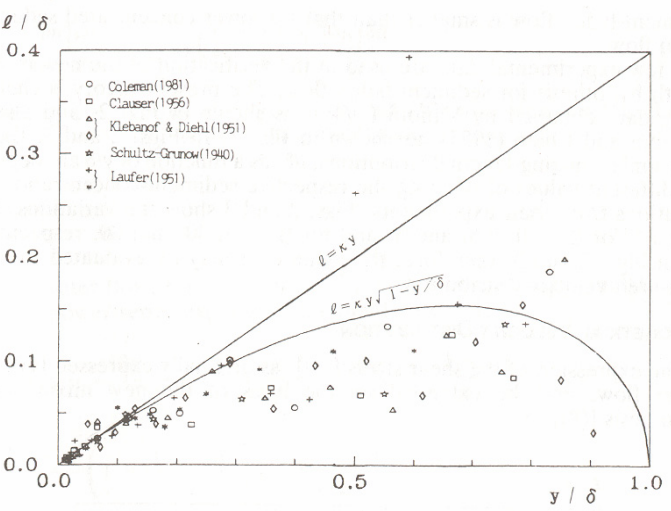

Fig.5 Comparison of vertical mixing length profile (20) with experimental data.

a) Theoretical profile $l(y)$ (curves) given by equation (20). solid line $c_{1}=1$; dash-dotted line: $c_{1}=2$; dotted line: $\mathrm{c}_{1}=4$; dashed line: equation (1).

b) Comparison between measurements (symbols) and the two roughness-independent mixing length equations (1) and (8), figure from ${ }^{15)}$.

length profiles from equations (1) and (8) which are roughness-independent. Fig. 5 shows that our mixing length profile (for a smooth wall) approaches the experimental values. Our proposed profile approaches more experimental data by increasing the value of the parameter $\mathrm{c}_{1}$.

\section{(2) The mean velocity profile}

Equation (19) provides a non-logarithmic velocity profile, since equations (16) and (19) give:

$$
\frac{d U}{d y}=\sqrt{C_{v}} \frac{\sqrt{k}}{l}=\frac{U_{f} \exp \left(-c_{1} \frac{y}{h}\right)}{\kappa\left[\frac{h}{c_{1}}-\left(\frac{h}{c_{1}}-y_{0}\right) \exp \left(-\frac{c_{1}\left(y-y_{0}\right)}{h}\right)\right]}
$$

with: $U_{a}=\frac{\kappa U}{U_{f}}, \xi=\frac{c_{1} y}{h}$ and $\xi_{0}=\frac{c_{1} y_{0}}{h}$, we write equation (30) as:

$$
\frac{d U_{a}}{d \xi}=\frac{\exp (-\xi)}{\left[1-\left(1-\xi_{0}\right) \exp \left(-\left(\xi-\xi_{0}\right)\right)\right]}
$$




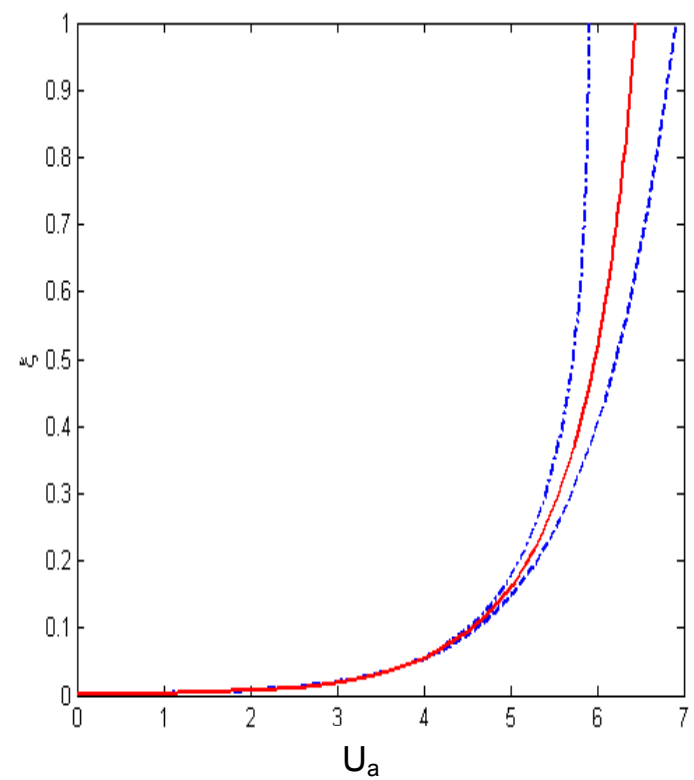

Fig.6 Comparison between the different mean velocity profiles. Solid line: equation (31); dashed line: logarithmic profile (equation 32); dash-dotted line: equation (33); for a relative roughness $\xi_{0}=0,001$.

We write therefore the logarithmic profile as:

$$
U_{a}=\ln (\xi)-\ln \left(\xi_{0}\right)
$$

and equation (11) in the form:

$$
\frac{d U_{a}}{d \xi}=\frac{(1-\xi)}{\xi}
$$

For equations (32) and (33), $\mathrm{c}_{1}=1$.

Fig. 6 presents a comparison between velocity profiles obtained respectively from (31), (32) and (33).

In order to validate the velocity profile obtained from our proposed mixing length equation, we analyze (Fig. 7) the velocity profile obtained from (30) by comparison with experimental data ${ }^{8)}$. Fig. 7 shows that the mean velocity profile computed with our mixing length equation (solid line) is more accurate than the logarithmic velocity profile (dash-dotted line).

\section{(3) Application of the time-dependent equation to wave boundary layers}

In coastal engineering, to improve the prediction of near shore sediment transport, we need to improve our understanding of the physical processes which occur in the wave or oscillatory boundary layer.

In this section, we apply our proposed timedependent equation to a turbulent oscillatory flow. The rough turbulent oscillating flow, is independent of the Reynolds number and depends

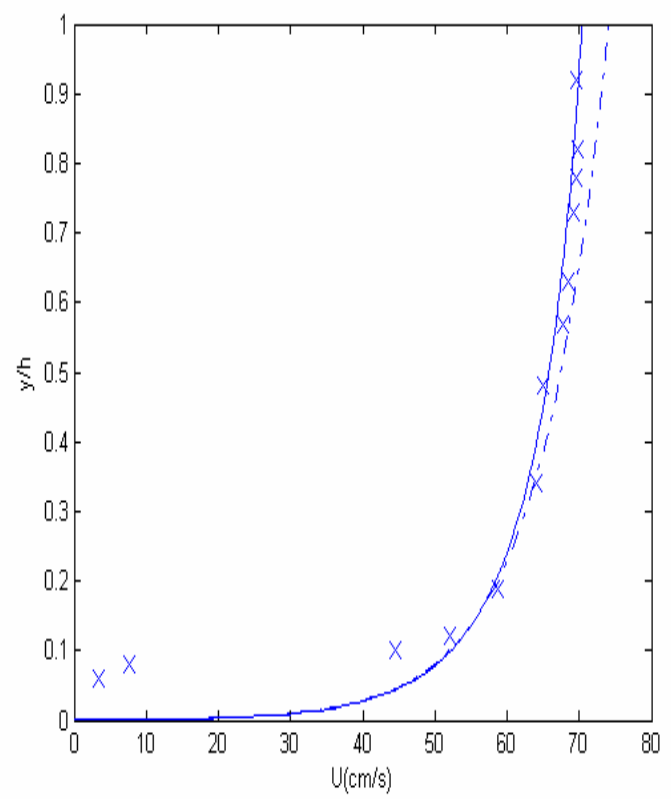

a)

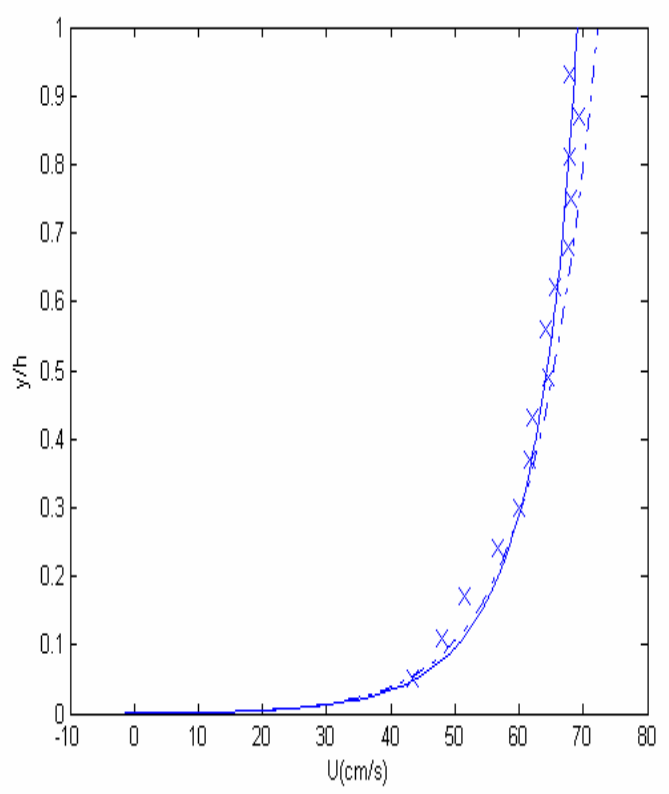

b)

Fig.7 Vertical distribution of mean flow velocity. Dashdotted line: logarithmic profile; solid line: obtained from equation (30) with $\mathrm{c}_{1}=1$; symbols: experimental data $^{8}$. a) profile 2: $\mathrm{y}_{0}=0.062 \mathrm{~cm} ; \mathrm{h}=145 \mathrm{~cm} ; \mathrm{U}_{\mathrm{f}}=$ $3.82 \mathrm{~cm} / \mathrm{s}$. b) profile 4: $\mathrm{y}_{0}=0.113 \mathrm{~cm} ; \mathrm{h}=164.5 \mathrm{~cm} ; \mathrm{U}_{\mathrm{f}}$ $=3.97 \mathrm{~cm} / \mathrm{s}$; values of $y_{0}, h$ and $U_{f}$ are from ${ }^{8}$.

only on the amplitude to bottom roughness ratio $a / k_{N}$, where $a=U_{0 m} / \omega$ is the free stream amplitude, $\omega$ the angular frequency of oscillation (Fig. 8.a)); $k_{N}$ is the equivalent Nikuradse roughness. As we can see in Fig. 8.b), the boundary layer thickness increases over the half wave period. The boundary layer thickness can be calculated simply by the von Kármán's momentum integral method. Fig. 8. c) shows that the mixing 
length computed from equation (27) also increases over the half wave period, since equation (27) is in the form $l \approx \delta$. In fact, when the two last terms in (27) becomes smaller than the first term, we find: $l=\kappa \delta$. This equation, which confirms the hypothesis (2), shows that an increase in the boundary layer thickness implies an increase in the mixing length. This figure shows that in oscillatory boundary layers, at the particular moment when the flow velocity approaches zero (flow reversal), there is an instability. In fact, the largest eddies, which are limited by the boundary layer thickness, grow in size during the half wave cycle and break up at the end of the half period.

In Fig. 8.d), the eddy viscosity is obtained with the time-dependent model (24), which consists of inserting our turbulent kinetic energy (26) and the proposed mixing length (27) into equation (4). In addition to the main peak, which is related to the maximum value of the friction velocity, Fig. 8.d) shows a second peak near flow reversal. These results demonstrate that our time-dependent eddy viscosity model is able to predict a sudden increase of the eddy viscosity near flow reversal. The $k-\varepsilon$ mode ${ }^{17,18)}$ also shows a sudden increase in the eddy viscosity at flow reversal. This is possible because it allows the variation of the mixing length scale in time, which is carried out by solving the $\varepsilon$ equation. Savioli and Justesen ${ }^{17)}$ and Malarkey et al. $^{18)}$ applied the $k-\varepsilon$ model in suspended sediment concentration modeling under oscillatory flows. They used the sudden increase in eddy viscosity near flow reversal in order to simulate the additional peak in suspended sediment concentration around flow reversal which was measured in different experimental studies ${ }^{19)}$.

Indeed, in oscillatory boundary layers with sediments, observations show an ejection of sand around flow reversal (Fig. 9).

In oscillatory flows, when the flow velocity approaches zero, experiments over a plane bed with sand show an extra entrainment. This phenomenon, which is associated to convective events due to boundary layer instability, seems to be related to rapid exchange of momentum and therefore a sudden increase in the eddy viscosity. This phenomenon was also associated ${ }^{17)}$ to a breakup of the boundary layer resulting in a burst.

Fig. 10 shows a comparison between our analytical model (24) and the $k-\varepsilon$ model $^{18)}$ for $a / k_{N}=144$ at two relative positions.
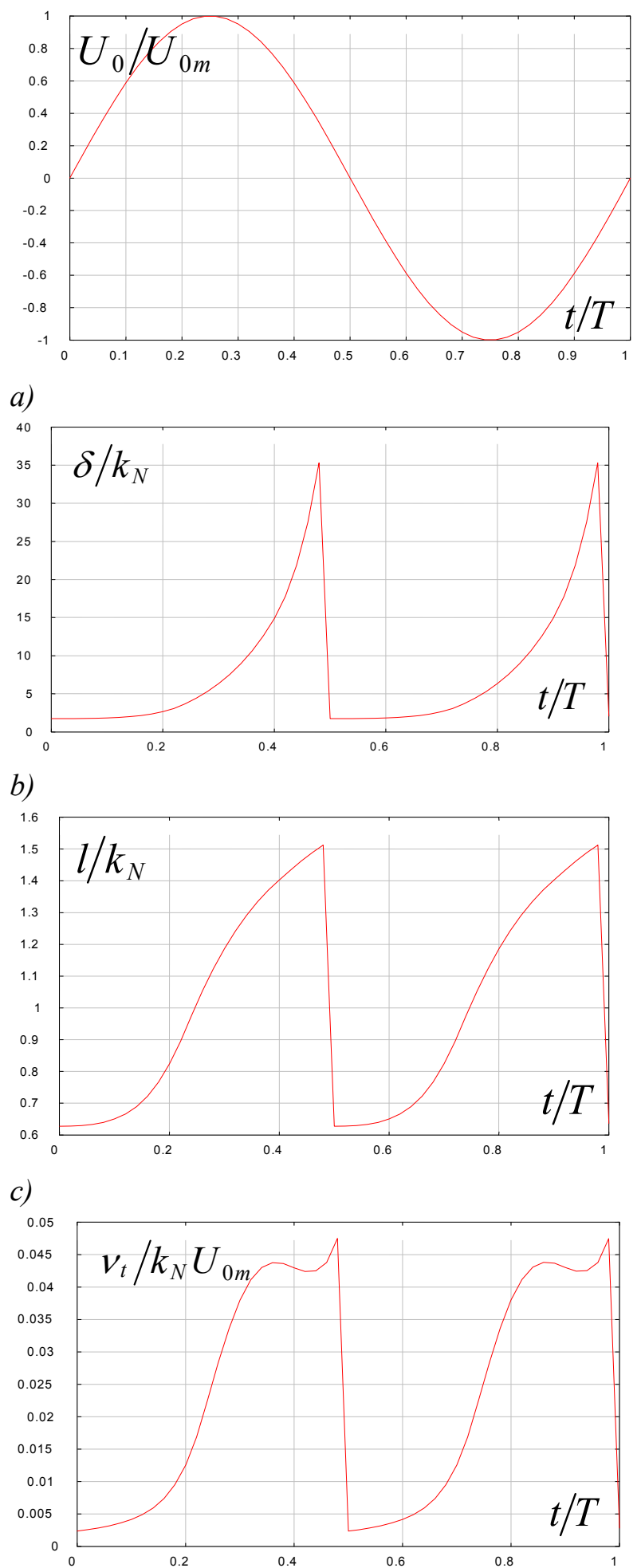

d)

Fig.8 Dimensionless free stream velocity, boundary layer thickness, mixing length and eddy viscosity for an oscillatory flow over one period ( $\left.a / k_{N}=144\right)$. Dimensionless: a) free stream velocity; b) boundary layer thickness; c) mixing length (27) at a relative position $y / k_{N}=4$; and d) eddy viscosity from our time-dependent model (24), at a relative position $y / k_{N}=4$. 


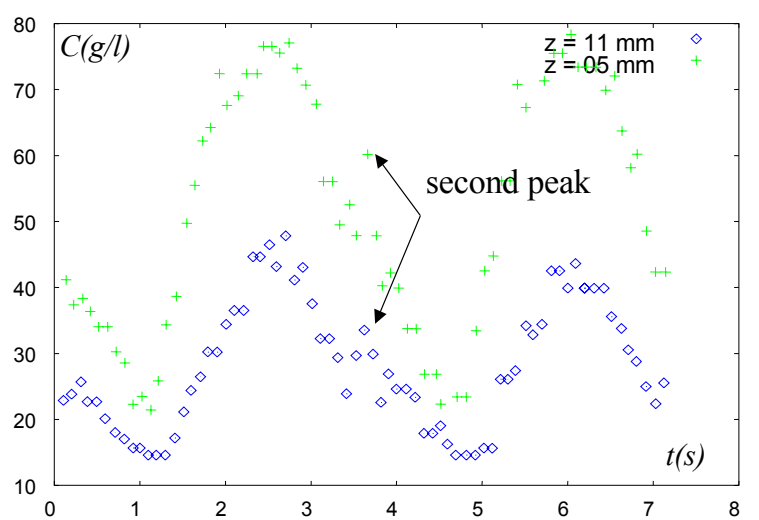

a)

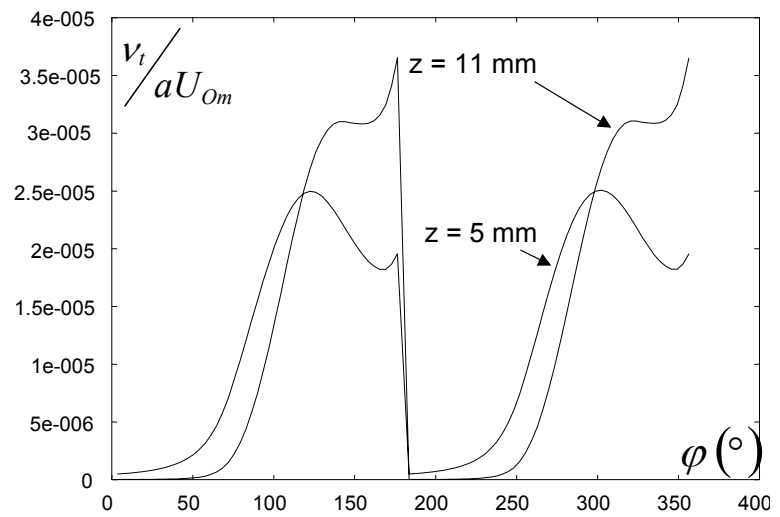

b)

Fig.9. Intra-wave (a) sediment concentrations, measurements $^{19)}$ and (b) eddy viscosity (present model) for two distances from the bed.

Since the flow is symmetric over the wave period (Fig. 8 and 9), we present only results over the half cycle. In Fig. 10, even if our curves follow the $k-\varepsilon$ model's results tendency, there is a difference. It is important to specify that the results depend directly on boundary layer thickness values. Since our model computes each half period independently, it does not take into account the effect of the first part of the period on the second part. The imperfection can be also explained by our two assumptions namely the similarity hypothesis and the local equilibrium, which in oscillatory flows could be not completely satisfying.

In oscillatory flows, velocity profiles are timedependent (Fig. 11). In order to obtain an accurate velocity profile in oscillatory boundary layers, it is important to consider a time-dependent friction velocity and boundary layer thickness:

$$
\frac{d U(y, t)}{d y}=\frac{U_{f}(t) \exp \left(-c_{1} \frac{y}{\delta(t)}\right)}{\kappa\left[\frac{\delta(t)}{c_{1}}-\left(\frac{\delta(t)}{c_{1}}-y_{0}\right) \exp \left(-\frac{c_{1}\left(y-y_{0}\right)}{\delta(t)}\right)\right]}
$$

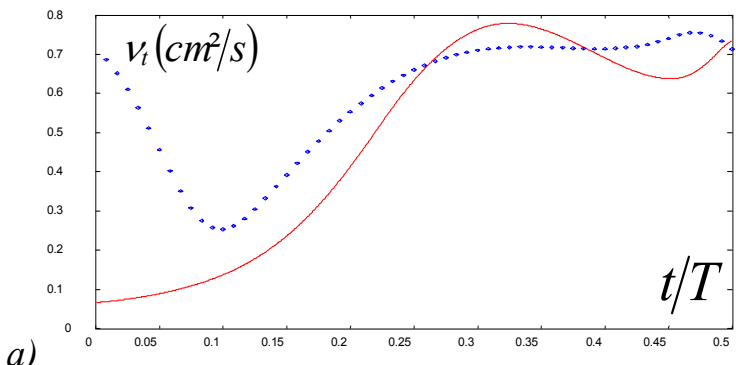

b)

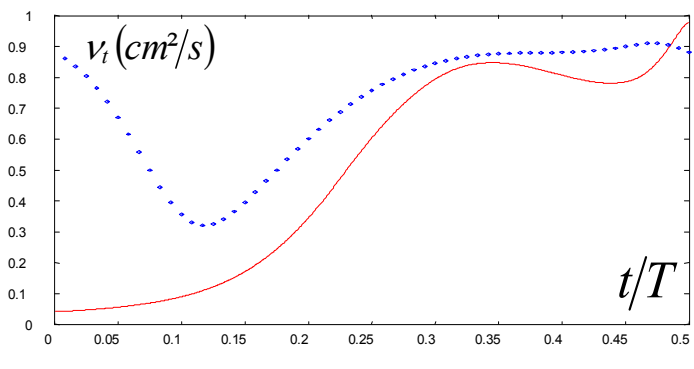

Fig.10 Time-dependant eddy viscosity, comparison between our model (curve) and the $\mathrm{k}-\varepsilon$ model (points) for $a / k_{N}=144$ at relative positions: a) $y / k_{N}=6$; b) $y / k_{N}=8$.

As represented in Fig. 11 and 12, the boundary layer thickness increases over the half-wave period.

Fig. 13 confirms that the use of the adequate boundary layer thickness $\delta$ (solid line) allows obtaining a more accurate velocity profile. The velocity profile for the phase $\pi / 4$, is computed (34) with the boundary layer thickness $\delta$ of the same phase (solid line) and with a boundary layer thickness $\delta$ of another phase (for example, we took $\delta$ for $(3 \pi) / 4$, dashed line). This figure shows that it is important to take the adequate $\delta$ for each phase and therefore a time-dependent boundary layer thickness in order to compute all phases.

\section{CONCLUSIONS}

In this paper, we proposed a roughness and time dependent equation for the mixing length. This mixing length equation is based on an extension of von Kármán's similarity hypothesis with an algebraic equation for the turbulent kinetic energy. From experimental data and a demonstration in equilibrium flows from the modeled $k$-equation, the vertical profile of turbulent kinetic energy $k$ is represented by an exponential function, which decreases with height. Using this shape for $k$, the integration of the equation based on the extension of von Kármán's similarity hypothesis gives our algebraic roughness and time dependent mixing length equation. 

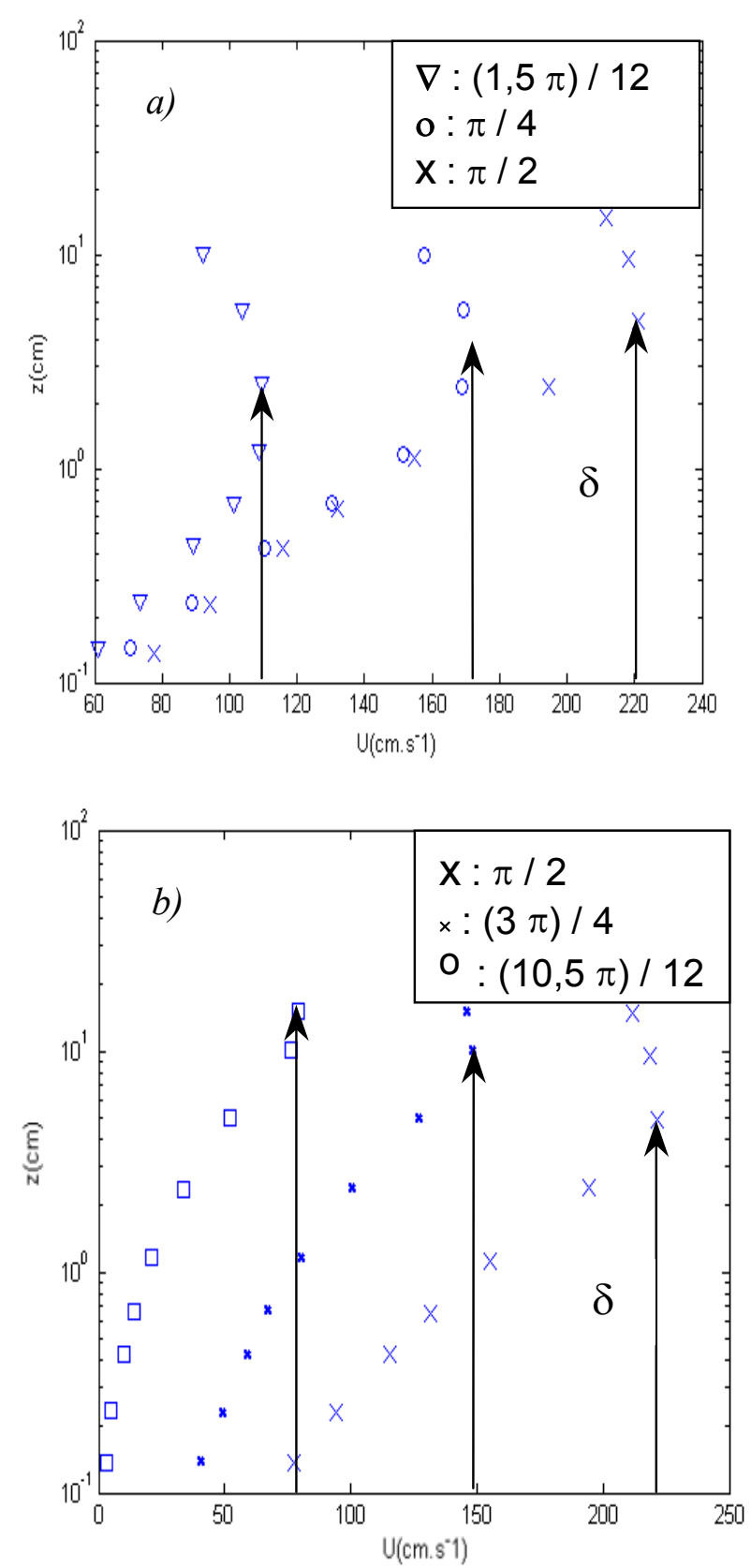

Fig.11 Time-dependent mean velocity profiles, symbols: measurements ${ }^{20)}$, the boundary layer thickness $\delta$ is represented for each phase $(\pi / 2$ correspond to the maximum value of the free stream velocity); a) for phases : $(1,5 \pi) / 12, \pi / 4, \pi / 2$; and $b)$ for phases : $\pi / 2$, $(3 \pi) / 4,(10,5 \pi) / 12$.

Our vertical mixing length profile, which is different from Prandtl's profile, approaches the experimental data well. We noticed that our mixing length increases more slowly with y. Moreover, the slope at the origin decreases with the roughness $y_{0}$; we introduced therefore an effective kappa $\kappa^{*}$. Even near the wall, our profile is different from Prandtl's profile. They will be identical only near smooth walls, only here $\kappa^{*} \approx \kappa$ and $l(y) \approx \kappa \cdot y$.

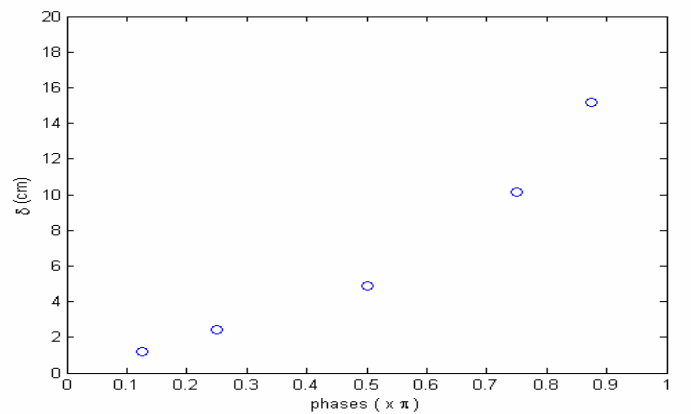

Fig.12 Time-dependent boundary layer thickness $\delta$ over the half wave period, obtained from mean velocity profiles ${ }^{20)}$.

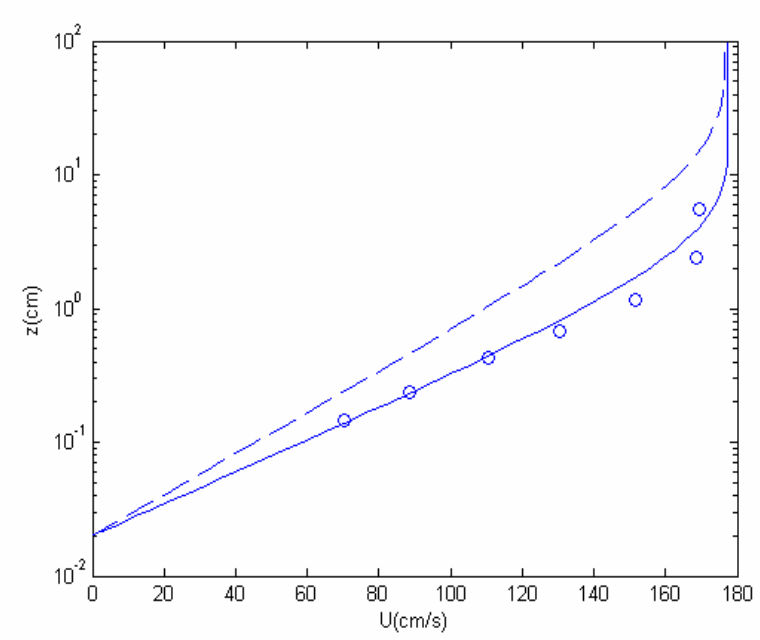

Fig.13 Velocity profile computed from equation (34), with two boundary layer thickness. symbols: experimental data for the phase $\pi / 4$; Solid line: computed from equation (34) with the boundary layer thickness of $\pi / 4$; dashed line: computed from equation (34) with the boundary layer thickness of $(3 \pi) / 4$.

Comparisons with experimental data show that the mean velocity profile computed with our mixing length equation is more accurate than the logarithmic velocity profile.

In order to validate our time-dependent mixing length equation, we applied it to a turbulent oscillatory flow. The results show, like the $k-\varepsilon$ model, that our time-dependent eddy viscosity model predicts a sudden increase of the eddy viscosity near flow reversal. This second peak in eddy viscosity allows simulating a measured extra entrainment (ejection) of sand around flow reversal in oscillatory flows over plane sandy beds. The comparison between our algebraic model and the $k-\varepsilon$ model shows that our model represents well the increase in the eddy viscosity near flow reversal. The difference, in the time-dependent curves, can be explained by the two model assumptions, namely the similarity hypothesis and the local equilibrium assumption. We have seen 
finally that in order to obtain an accurate velocity profile in oscillatory boundary layers, it is important to consider a time-dependent boundary layer thickness.

ACKNOWLEDGMENT: The author would like to thank the anonymous reviewers for their helpful comments in improving this paper.

\section{REFERENCES}

1) Escudier, M.P.: The distribution of mixing-length in turbulent flows near walls. Imp. Coll. Heat Transfer Section Rep. TWF/TN/1, 1966.

2) Absi, R.: Discussion of one-dimensional wave bottom boundary layer model comparison: specific eddy viscosity and turbulence closure models, ASCE J. Wtrwy. Port Coast. Oc. Eng., 132(2), pp. 139-141, 2006.

3) von Kármán, Th.: Mechanische Ähnlichkeit und Turbulenz, Nach. Ges. Wiss. Gottingen, Math.-Phys. Klasse, 58, 1930.

4) Nezu, I. and Nakagawa, H.: Turbulence in open-channel flows. A.A. Balkema, Ed. Rotterdam, The Netherlands, 1993.

5) Song, T., Graf, W.H. and Lemmin, U.: Uniform Flow in Open Channels with Movable Gravel Bed, J. Hydr. Res., 32(6), pp. 861-876, 1994.

6) Wijetunge, J.J. and Sleath, J.F.A.: Effects of sediment transport on bed friction and turbulence, ASCE J. Wtrwy. Port Coast. Oc. Eng., 124(4), pp. 172-178, 1998.

7) Absi, R.: Time-dependent eddy viscosity models for wave boundary layers, Proc. 27 $7^{\text {th }}$ Int. Conf. on Coastal Engineering, Sydney, ASCE, Billy. L. Edge (Ed.), ASCE Press, Reston VA, pp. 1268-1281, 2000.

8) Sukhodolov A., Thiele M. and Bungartz H.: Turbulence structure in a river reach with sand bed, Water Resour. Res., 34, pp. 1317-1334, 1998.

9) Absi, R.: Wave boundary layer instability near flow reversal, Proc. $28^{\text {th }}$ Int. Conf. on Coastal Engineering, Cardiff, ASCE,
Jane McKee Smith (Ed.), World Scientific, Singapore, pp. 532-544, 2002.

10) Absi, R.: A simple mixing length equation from an extension of von Kármán's similarity hypothesis, In Book of Abstracts of $5^{\text {th }}$ EUROMECH Fluid Mechanics Conference, p. 307, 2003.

11) Fredsøe, J., Andersen, O.H. and Silberg, S.: Distribution of suspended sediment in large waves. ASCE J. Wtrwy. Port Coast. Oc. Eng., 111(6), pp. 1041-1059, 1985.

12) Jensen, B.L., Sumer, B.M. and Fredsøe, J.: Turbulent oscillatory boundary layers at high Reynolds numbers. $J$. Fluid Mech., 206, pp. 265-297, 1989.

13) Absi, R.: Calibration of Businger-Arya type of eddy viscosity model's parameters: discussion, ASCE J. Wtrwy. Port Coast. Oc. Eng., 126(2), pp. 108-109, 2000.

14) Sleath, J.F.A.: Turbulent oscillatory flow over rough beds. J. Fluid Mech., 182, pp. 369-409, 1987.

15) Umeyama, M. and Gerritsen, F.: Velocity distribution in uniform sediment-laden flow, ASCE J. Hyd. Eng., 118(2), pp. 229-245, 1992.

16) Absi, R. and Marchandon, S.: Longueur de mélange et diffusion turbulente des sédiments, Actes des VIIIème journées nationales 'Génie côtier - Génie civil', UTC, Compiègne, pp. 279-287, 2004.

17) Savioli, J. and Justesen, P.: Sediment in oscillatory flows over plane bed, J. Hydr. Res., 35(2), pp. 177-190, 1997.

18) Malarkey, J., Davies, A.G. and Li, Z.: A simple model of oscillatory sheet flow. Proc. $28^{\text {th }}$ Int. Conf. on Coastal Engineering, Cardiff, ASCE, Jane McKee Smith (Ed.), World Scientific, Singapore, pp. 2491-2503, 2002.

19) Ribberink, J.S. and Al-Salem, A.A.: Sheet flow and suspension of sand in oscillatory boundary layers. Coast. Eng., Elsevier, 25, pp. 205-225, 1995.

20) Sumer, B.M., Jensen, B.L. and Fredsøe, J.: Turbulence in oscillatory boundary layers. Advances in Turbulence. Advances in turbulence, eds., G. Comte-Bellot and J. Mathieu, Springer, Berlin, pp. 556-567, 1987.

(Received June 21, 2005) 